\title{
Intracorporeal versus extracorporeal anastomosis in laparoscopic right hemicolectomy - single center experience
}

\author{
Andrzej P. Kwiatkowski ${ }^{1}$, Gabriela Stępińska ${ }^{2}$, Edward Stanowski ${ }^{1}$, Krzysztof Paśnik ${ }^{1}$, Michal R. Janik ${ }^{1}$ \\ ${ }^{1}$ Department of General, Oncological, Metabolic and Thoracic Surgery, Military Institute of Medicine, Warsaw, Poland \\ ${ }^{2}$ First Faculty of Medicine, Medical University of Warsaw, Warsaw, Poland
}

Videosurgery Miniinv 2019; 14 (3): 381-386

DOI: https://doi.org/10.5114/wiitm.2019.81725

\begin{abstract}
Introduction: Nowadays laparoscopic right hemicolectomy is widely accepted as the standard of care for benign and malignant colon disease. There are wide variations among laparoscopic techniques. One of the most discussed topics is the ileocolic anastomosis. There are two different techniques: intracorporeal anastomosis (IA) and extracorporeal anastomosis (EA).

Aim: To compare short-term outcomes of performing intracorporeal versus extracorporeal anastomosis in laparoscopic right hemicolectomy.

Material and methods: A retrospective chart review was performed of 92 consecutive patients who underwent laparoscopic right hemicolectomy, with either an IA or EA, from January 2013 to December 2016.

Results: Eighty-five patients were included in the analysis. There were 53 males and 32 females with a mean age of $67.1 \pm 13.2$ years. Mean body mass index (BMI) was $27.7 \pm 4.8 \mathrm{~kg} / \mathrm{m}^{2}$. An intracorporeal anastomosis was performed in 51 patients, while an extracorporeal anastomosis was performed in 34. The duration of operations was significantly longer when intracorporeal anastomosis was performed, taking $154 \pm 58$ min compared to $95 \pm 34$ min $(p<0.001)$, in the extracorporeal group. No mortality was observed in the IA group. The postoperative mortality in the EA group was $8.8 \%(p=0.060)$. The rate of reoperation in the intracorporeal anastomosis group was $7.8 \%$, whereas in the extracorporeal anastomosis group it was $14.7 \%(p=0.474)$. Length of hospital stay in the IA group was shorter in comparison to the EA group ( $5.3 \pm 3.7$ vs. $11.2 \pm 19.8$ days, $p=0.022)$.

Conclusions: Our results are encouraging to consider the intracorporeal approach as the better way to fashion the anastomosis after laparoscopic right hemicolectomy.
\end{abstract}

Key words: intracorporeal, extracorporeal, anastomosis, laparoscopic right hemicolectomy, complications, outcomes.

\section{Introduction}

Since the introduction of mini-invasive colorectal surgery many large randomized trials have demonstrated oncologically equivalent outcomes for laparoscopic and open colon resection [1]. Nowadays laparoscopic right hemicolectomy is widely accepted as the standard of care for benign and malignant colon disease $[2,3]$. Laparoscopy has many bene- fits including improved recovery, reduced analgesia needs, lower rate of surgical site infection and shorter hospital stay. There are wide variations among laparoscopic techniques, including the approach for bowel mobilization, anastomosis, mesentery defect closure, etc. One of the most discussed topics is the ileocolic anastomosis. There are two different techniques: intracorporeal anastomosis (IA) and extracorporeal anastomosis (EA). The advantages of IA

\section{Address for correspondence}

Andrzej P. Kwiatkowski MD, PhD, Department of General, Oncological, Metabolic and Thoracic Surgery, Military Institute of Medicine,

128 Szaserów St, 04-141 Warsaw, Poland, phone: +48 791017093, e-mail: andkwiatkowski@poczta.onet.pl 
are avoidance of traction of the mesentery during extraction of the bowel, the free choice of the site of specimen extraction, full visualization of every step of creation of the anastomosis, and easy management in obese patients. The disadvantages of IA are: necessity for advanced laparoscopic suturing skills, longer learning curve, and possible bowel contaminations. It is currently debated which of the two anastomosis technique is more suitable.

\section{Aim}

The purpose of this study was to compare shortterm outcomes of performing intracorporeal versus extracorporeal anastomosis in laparoscopic right hemicolectomy

\section{Material and methods}

A retrospective chart review was performed of 92 consecutive patients who underwent laparoscopic right hemicolectomy, with either an IA or EA from January 2013 to December 2016. All of the procedures were performed by experienced surgical oncologists and the type of anastomosis was the surgeon's preference. Patients who underwent conversion to open surgery during the procedure or had radiotherapy prior to surgery were excluded. The demographic data were collected, including information related to sex, age, body mass index (BMI), American Society of Anesthesiologists (ASA) score, diagnostic group (benign/malignant), and the following previous abdominal procedures: appendectomy, cholecystectomy, hernia, hysterectomy, sigmoidectomy and others. Surgical variables included operative time, postoperative mortality and rates of complications, conversion, reoperation, length of hospital stay and readmission within 30 days. Major complications included anastomotic leak and bleeding. Other complications included: wound infection, ileus and bowel obstruction, dehiscence, and strangulation of inguinal hernia. Pathologic data included the following: anatomopathological diagnosis, TNM stage and number of removed lymph nodes (isolated and positive).

\section{Surgical technique}

The patient was placed in a supine position, the surgeon was standing on the left, assistant next to the surgeon on his left side. Three trocars were used: one $10 \mathrm{~mm}$, a $5 \mathrm{~cm}$ to the left of the umbilicus, one
$12 \mathrm{~mm}$ in the left epigastric region, and one $5 \mathrm{~mm}$ in the midline $5 \mathrm{~cm}$ over the pubis. The ileocolic and right branch of media colic vessels were identified and dissected after application of hemostatic clips or a bipolar sealing device. The colon was mobilized systematically via a medial-to-lateral approach. A side-to-side isoperistalsis anastomosis was created with a $60 \mathrm{~mm}$ endostapler and the enterotomy was closed with continuous double layer suture. The mesenteric defect was closed by two Z sutures. The specimen was extracted through a Pfannenstiel incision. The incision for specimen extraction was protected in all cases with an isolation device. Endobags were not used. In the case of EA the position of the patient and operational team was the same. Four trocars were used: the first $10 \mathrm{~mm}$ in the umbilicus, the second $10 \mathrm{~mm}$ in the left epigastric region, a $5 \mathrm{~mm}$ trocar in the left iliac fossa, a $5 \mathrm{~mm}$ trocar in the right iliac fossa. The ileocolic and right branches of media colic vessels were identified and dissected after application of hemostatic clips. The colon was mobilized systematically via a medial-to-lateral approach. The minilaparotomy was performed by right paramedial incision. The incision was protected in every case with an isolation device. A side-to side antiperistalsis fully stapled anastomosis was performed with a $55 \mathrm{~mm}$ GIA stapler. The mesenteric defect was not closed.

\section{Statistical analysis}

Data analysis was performed using the SAS software, University Edition (SAS Institute Inc., Cary, NC, USA). Comparisons of continuous parameters were performed by Student's test or the Mann-Whitney test, according to data distribution. The $\chi^{2}$ test or Fisher's exact test for small samples was used to compare categorical variables. All $p$-values are two-sided, and statistical significance is defined as $p \leq 0.05$.

\section{Results}

There were 92 patients who underwent laparoscopic right hemicolectomy in the period from January 2013 to December 2016. Seven patients were excluded -6 due to conversion to an open procedure and 1 patient was excluded due to radiotherapy prior to surgery. Finally, 85 patients were included in the analysis. There were 53 males and 32 females with a mean age of $67.1 \pm 13.2$ years. Mean BMI was 27.7 
$\pm 4.8 \mathrm{~kg} / \mathrm{m}^{2}$. Twenty-eight patients had a history of at least one prior abdominal operation. Indications for surgery included neoplasm in 74 patients, and benign pathology in the rest. An intracorporeal anastomosis was performed in 51 patients, while an extracorporeal anastomosis was performed in 34. The groups were comparable in terms of demographic variables, which are shown in Table I. The surgical variables are shown in Table II. The duration of operations was significantly longer when intracorporeal anastomosis was performed, taking $154 \pm 58$ min compared to $95 \pm 34$ min in the extracorporeal group. No mortality was observed in the IA group. The postoperative mortality in the EA group was $8.8 \%$, due to cardiorespiratory complications. Although statistical significance was not achieved, we consider the result clinically significant. We did not observe anastomotic leak in the IA group but the incidence of anastomotic leak in the EA group was $8.8 \%$. Although statistical significance was not achieved, we consider the result clinically significant. Bleeding occurred in 1 patient in the IA group (2.0\%), and in 2 cases in the EA group (5.9\%). Other complications were clinically less common in the IA group (7.8\%) compared to the EA group (23.5\%). Reoperation was required in 9 cases in total. The rate of reoperation in the intracorporeal anastomosis group was 7.8\%, whereas in the extracorporeal anastomosis group it was $14.7 \%$. Of these cases, two underwent surgery due to internal bleeding, two due to anastomosis dehiscence and two due to wound dehiscence and evisceration. The rest of indications for reoperations included incarcerated hernia, intestinal fistula and postoperative ileus. Length of hospital stay in the IA

Table I. Baseline characteristics

\begin{tabular}{|lccc|}
\hline Parameter & IE $(n=51)$ & EA $(n=34)$ & $P$-value \\
\hline Age $($ range $)[$ years] & $66.2(35-87)$ & $68.6(28-85)$ & 0.229 \\
\hline Sex $(\mathrm{M} / \mathrm{F}), n(\%)$ & $31(60.8 \%) / 20(39.2 \%)$ & $22(64.7 \%) / 12(35.3 \%)$ & 0.820 \\
\hline BMI (range) $\left[\mathrm{kg} / \mathrm{m}^{2}\right]$ & $27.9(20.1-39.0)$ & $27.6(18.6-41.9)$ & 0.668 \\
\hline ASA (I: II : III : IV) & $5(9.8 \%): 33(64.7 \%): 13(25.5 \%): 0$ & $3(9.1 \%): 22(66.7 \%): 7(8.3 \%): 1(1.2 \%)$ & 0.739 \\
\hline Previous abdominal surgery & $17(33.0 \%)$ & $11(32.3 \%)$ & 1.000 \\
\hline $\begin{array}{l}\text { Indication for surgery: } \\
\begin{array}{l}\text { Malignant/benign } \\
\text { pathology }\end{array}\end{array}$ & $43(84.3 \%) / 8(15.7 \%)$ & $28(82.4 \%) / 6(17.6 \%)$ & \\
\hline
\end{tabular}

Table II. Surgical outcomes

\begin{tabular}{|lccc|}
\hline Parameter & $\mathrm{IE}(n=51)$ & $\mathrm{EA}(n=34)$ & $P$-value \\
\hline Operative time (range) [min] & $154(55-335)$ & $95(30-210)$ & $<0.001$ \\
\hline Number of lymph nodes removed (range) & $14.3(0-31)$ & $9.7(2-26)$ & 0.117 \\
\hline Length of hospital stay, mean \pm SD (range); median & $5.3 \pm 3.7(2-18) ; 4.0$ & $11.2 \pm 19.8(3-105) ; 5.0$ & 0.022 \\
\hline Rate of complications: & $5(9.8 \%)$ & $9(26.4 \%)$ & 0.071 \\
\hline Bleeding & $1(2 \%)$ & $2(5.9 \%)$ & 0.561 \\
\hline Anastomotic leak & 0 & $3(8.8 \%)$ & 0.060 \\
\hline Abdominal abscess & 0 & $1(3 \%)$ & 0.400 \\
\hline Other & $4(7.8 \%)$ & $8(23.5 \%)$ & 0.058 \\
\hline Rate of reoperation & $4(7.8 \%)$ & $5(14.7 \%)$ & 0.474 \\
\hline Readmission & $0(0 \%)$ & $1(2.9 \%)$ & 0.400 \\
\hline Mortality & 0 & $3(8.8 \%)$ & 0.060 \\
\hline
\end{tabular}


group was shorter in comparison to the EA group (5.3 \pm 3.7 vs. $11.2 \pm 19.8$ days, $p=0.022)$. The majority of complications occurred during index hospitalization and 30-day readmission rates were comparable between IA and EA groups (0.0\% vs. 2.9\%). Pathological outcomes are presented in Table III.

\section{Discussion}

Mini-invasive colon surgery is increasingly regarded as the gold standard for benign and malignant lesions. Short-term advantages of the laparoscopic approach for colon resection are well recognized [4].
The safety and oncological radicality of mini-invasive colectomy have been proven by many randomized, controlled trials (COLOR, CLASSIC, BARCELONA, COST) [5-8]. Each type of colon resection has his own level of difficulty. Jamali et al. showed that laparoscopic right hemicolectomy with extracorporeal anastomosis is more technically demanding than sigmoidectomy and its difficulty is on the rise when anastomosis is managed totally intracorporeally [9]. Thus, this is a reason why the majority of surgeons still choose to perform EA [10,11]. Although rapid implementation of miniinvasive technique in

Table III. Pathological findings. TNM staging was applied for patients with diagnosis of adenocarcinoma

\begin{tabular}{|c|c|c|c|}
\hline Characteristics & $\mathrm{IE}(n=51)$ & $\mathrm{EA}(n=34)$ & $P$-value \\
\hline \multicolumn{3}{|l|}{ Pathological diagnosis: } & 0.382 \\
\hline Adenocarcinoma & $43(84.3 \%)$ & $28(82.4 \%)$ & \\
\hline Adenoma & $5(9.8 \%)$ & $4(11.8 \%)$ & \\
\hline Appendicitis & $1(1.96 \%)$ & 0 & \\
\hline Colitis chronica & 0 & $1(2.9 \%)$ & \\
\hline Crohn's disease & $1(1.96 \%)$ & 0 & \\
\hline GIST & $1(1.96 \%)$ & 0 & \\
\hline Neuroendocrine tumor & 0 & $1(2.9 \%)$ & \\
\hline \multicolumn{3}{|l|}{ T staging: } & 0.645 \\
\hline $\mathrm{T} 1$ & $2(4.6 \%)$ & $2(7.1 \%)$ & \\
\hline $\mathrm{T} 2$ & 9 (20.9\%) & 5 (17.9\%) & \\
\hline $\mathrm{T} 3$ & $29(67.4 \%)$ & $18(64.3 \%)$ & \\
\hline $\mathrm{T} 4$ & $3(7.0 \%)$ & $3(10.7 \%)$ & \\
\hline \multicolumn{3}{|l|}{ N staging: } & 0.352 \\
\hline NO & $23(53.5 \%)$ & $18(64.3 \%)$ & \\
\hline N1 & $10(23.3 \%)$ & $5(17.8 \%)$ & \\
\hline $\mathrm{N} 2$ & $10(23.3 \%)$ & $5(17.8 \%)$ & \\
\hline \multicolumn{3}{|l|}{ M staging: } & 1.000 \\
\hline MO & $34(79.1 \%)$ & $23(82.1 \%)$ & \\
\hline M1 & $9(20.9 \%)$ & $5(17.9 \%)$ & \\
\hline \multicolumn{3}{|l|}{ Grading: } & 0.872 \\
\hline G1 & $2(4.6 \%)$ & $2(7.1 \%)$ & \\
\hline $\mathrm{G} 2$ & $38(88.4 \%)$ & $24(85.7 \%)$ & \\
\hline G3 & $3(7.0 \%)$ & $2(7.14 \%)$ & \\
\hline G4 & 0 & 0 & \\
\hline
\end{tabular}


colorectal surgery is possible, the main obstacle for intracorporeal anastomosis is a need for advanced suturing and binding skills [12].

Many studies try to show which type of anastomosis offers more benefits. Still there are controversies. The meta-analysis by Feroci et al. showed no statistically significant differences in operating time, post-surgical complications, reoperations, number of isolated lymph nodes or mortality [13]. However, the meta-analysis performed by Milone et al. revealed that mini-invasive colon surgery with intracorporeal anastomosis was associated with a lower rate of postoperative complications. The main difference was consistently found for less severe class I and II [14]. Similar results were observed in our study. The length of hospital stay was shorter in the IA group in comparison to the EA group. It was caused by a lower rate of complications in the IA group. The leak rate in this group corresponds with the leak rate reported by other Polish group [15]. However, in the EA group we noted a high incidence of complication class III, especially anastomosis leak. In our opinion, this difference may have resulted from the different technical approach to anastomosis. In the EA group, it was fully stapled and antiperistalsis. It resulted in crossing the staple line and the tendency for twisting.

Incisional hernia is a common complication of both open and keyhole surgery. The incisional hernia is a common complication of both open and keyhole surgery. The initial enthusiasm for a lower rate of herniation after the mini-invasive approach was partly tempered. The main point of concern is the extraction site. The midline incision, which is most preferable for EA, is associated with the highest risk of herniation. Two prospective randomized trials showed almost the same rate of hernia for the midline incision despite of type of approach. The reported range in the study done by Winslow et al. was $24.3 \%$ versus $19.6 \%$ for laparoscopic versus open surgery respectively [16]. Although the trial done by Braga et al. showed lower risk of hernation in miniinvasive group $4.7 \%$ versus $8.9 \%$ in open group, the difference was not significant [17].

One of the main benefits of the IA approach is free choice of the site of mini-laparotomy. The study performed by DeSouza et al. analyzed the ideal extraction site for mini-invasive colorectal resection and they found that a Pfannenstiel incision was associated with the lowest rate of incisional hernia; therefore, it was their recommendations to use it for laparoscopic colorectal surgery wherever applicable [18]. Nevertheless, in their study, the Pfannenstiel incision was used mainly for anterior resection, while right colectomies were mainly performed using a mini midline laparotomy incision. Data suggest that the Pfannenstiel incision is superior to others in terms of hernia rate $[19,20]$. The size of specimen could also reduce the benefits of the laparoscopic approach, especially for an EA, but using the Pfannenstiel incision gives a clear advantage for pain control and hernia rate even in the case of greater length of the wound [21, 22].

One of the most important factors for increased level of difficulty of surgery is obesity [23]. The length of incision for extracorporeal anastomosis is often associated with the level of obesity. The reason for this is the thickness of abdomen wall and lower mobility of mesentery. The benefits of IA in this group of patients are more visible [24].

Additional benefits of IA are: mobilization of the bowel is performed under direct vision, with lack of traction on the mesentery. Therefore, it reduces the risk of mesenteric tears, bleeding and torsion of the mesentery. These advantages could result in enhanced recovery, decreased complication rate, less adhesion formation and improved long-term results $[25,26]$.

Some limitations of the study should be addressed. First, this is a retrospective analysis. Second, due to the small sample size there is a risk of Il type errors. Third, this study was not randomized. For these reasons we considered the $p$-value below 0.1 as clinically significant.

\section{Conclusions}

Our results are encouraging to consider the intracorporeal approach the better way to perform the anastomosis after laparoscopic right hemicolectomy. This study clearly provides the rationale for randomized clinical trials, which would be useful to give a definitive answer.

\section{Conflict of interest}

The authors declare no conflict of interest.

\section{References}

1. Lee SW. Laparoscopic procedures for colon and rectal cancer surgery. Clin Colon Rectal Surg 2009; 22: 218-24.

2. van Oostendorp S, Elfrink A, Borstlap W, et al. Intracorporeal versus extracorporeal anastomosis in right hemicolectomy: 
a systematic review and meta-analysis. Surg Endosc 2017; 31: 64-77.

3. Dulskas A, Samalavicius NE, Gupta RK, et al. Laparoscopic colorectal surgery for colorectal polyps: single institution experience. Videosurgery Miniinv 2015; 10: 73-8.

4. Veldkamp R, Kuhry E, Hop WCJ, et al. Laparoscopic surgery versus open surgery for colon cancer: short-term outcomes of a randomised trial. Lancet Oncol 2005; 6: 477-84.

5. Nelson H, Sargent DJ, Wieand HS, et al. A comparison of laparoscopically assisted and open colectomy for colon cancer. N Engl J Med 2004; 350: 2050-9.

6. Guillou PJ, Quirke P, Thorpe H, et al. Short-term endpoints of conventional versus laparoscopic-assisted surgery in patients with colorectal cancer (MRC CLASICC trial): multicentre, randomised controlled trial. Lancet 2005; 365: 1718-26.

7. Lacy AM, Delgado S, Castells A, et al. The long-term results of a randomized clinical trial of laparoscopy-assisted versus open surgery for colon cancer. Ann Surg 2008; 248: 1-7.

8. Fleshman J, Sargent DJ, Green E, et al. Laparoscopic colectomy for cancer is not inferior to open surgery based on 5-year data from the COST Study Group trial. Ann Surg 2007; 246: 654-5.

9. Jamali FR, Soweid AM, Dimassi H, et al. Evaluating the degree of difficulty of laparoscopic colorectal surgery. Arch Surg 2008; 143: 762-7.

10. Carnuccio P, Jimeno J, Pares D. Laparoscopic right colectomy: a systematic review and meta-analysis of observational studies comparing two types of anastomosis. Tech Coloproctol 2014; 18: 5-12.

11. Simorov A, Shaligram A, Shostrom V, et al. Laparoscopic colon resection trends in utilization and rate of conversion to open procedure: a national database review of academic medical centers. Ann Surg 2012; 256: 462-8.

12. Kwiatkowski AP, Stępińska G, Stanowski E, et al. Implementation of laparoscopic approach in colorectal surgery - a single center's experience. Videosurgery Miniinv 2018; 13: 27-32.

13. Feroci F, Lenzi E, Garzi A, et al. Intracorporeal versus extracorporeal anastomosis after laparoscopic right hemicolectomy for cancer: a systematic review and meta-analysis. Int J Colorectal Dis 2013; 28: 1177-86.

14. Milone M, Elmore U, Di Salvo E, et al. Intracorporeal versus extracorporeal anastomosis. Results from a multicentre comparative study on 512 right-sided colorectal cancers. Surg Endosc 2015; 29: 2314-20.

15. Wierdak M, Pisarska M, Kuśnierz-Cabala B, et al. Use of inflammatory markers in the early detection of infectious complications after laparoscopic colorectal cancer surgery with the ERAS protocol. Videosurgery Miniinv 2018; 13: 315-25.

16. Winslow ER, Fleshman JW, Birnbaum EH, et al. Wound complications of laparoscopic vs open colectomy. Surg Endosc 2002; 16: 1420-5.

17. Braga M, Frasson M, Vignali A, et al. Laparoscopic vs. open colectomy in cancer patients: long-term complications, quality of life, and survival. Dis Colon Rectum 2005; 48: 2217-23.

18. DeSouza A, Domajnko B, Park J, et al. Incisional hernia, midline versus low transverse incision: what is the ideal incision for specimen extraction and hand-assisted laparoscopy? Surg Endosc 2011; 25: 1031-6.
19. Lee L, Abou-Khalil M, Liberman S, et al. Incidence of incisional hernia in the specimen extraction site for laparoscopic colorectal surgery: systematic review and meta-analysis. Surg Endosc 2017; 31: 5083-93.

20. Benlice C, Stocchi L, Costedio MM, et al. Impact of the specific extraction-site location on the risk of incisional hernia after laparoscopic colorectal resection. Dis Colon Rectum 2016; 59: 743-50.

21. Kisielinski K, Conze J, Murken AH, et al. The Pfannenstiel or so called "bikini cut": still effective more than 100 years after first description. Hernia 2004; 8: 177-81.

22. Llaguna OH, Avgerinos D V, Lugo JZ, et al. Incidence and risk factors for the development of incisional hernia following elective laparoscopic versus open colon resections. Am J Surg 2010; 200: 265-9.

23. Rabasová M, Martínek L. Conversion risk factors in laparoscopic colorectal surgery. Videosurgery Miniinv 2012; 7: 240-5.

24. Vignali A, Elmore U, Lemma M, et al. Intracorporeal versus extracorporeal anastomoses following laparoscopic right colectomy in obese patients: a case-matched study. Dig Surg 2018; 35: 236-42.

25. Shapiro R, Keler U, Segev L, et al. Laparoscopic right hemicolectomy with intracorporeal anastomosis: short- and long-term benefits in comparison with extracorporeal anastomosis. Surg Endosc 2016; 30: 3823-9.

26. Bartels SAL, Vlug MS, Hollmann MW, et al. Small bowel obstruction, incisional hernia and survival after laparoscopic and open colonic resection (LAFA study). Br J Surg 2014; 101: 1153-9.

Received: 2.10.2018, accepted: 27.11.2018. 